\title{
A novel cell wall-anchored peptidoglycan hydrolase
(autolysin), IspC, essential for Listeria
monocytogenes virulence: genetic and proteomic
analysis \\ Correspondence \\ Min Lin \\ linm@inspection.gc.ca \\ Received 17 November 2007 \\ Revised 14 March 2008 \\ Accepted 25 March 2008 \\ Linru Wang ${ }^{1,2}$ and Min Lin ${ }^{1,2}$

\author{
${ }^{1}$ Animal Diseases Research Institute, Ottawa, ON K2H 8P9, Canada \\ ${ }^{2}$ Department of Biochemistry, Microbiology and Immunology, University of Ottawa, Ottawa, ON K1H \\ 8M5, Canada
}

\begin{abstract}
We have recently concluded that a Listeria monocytogenes $86 \mathrm{kDa}$ immunogenic surface protein, IspC, is a cell wall-anchored peptidoglycan hydrolase (autolysin), capable of degrading the cell wall peptidoglycan of the bacterium itself. To determine if this enzyme has any biological functions and/or plays a role in virulence, we in-frame-deleted the ispC gene from the $L$. monocytogenes chromosome. This $\Delta i s p C$ mutant exhibited complete abrogation of expression of IspC and displayed no defects in in vitro growth, colony and microscopic morphologies, or biochemical characteristics. Lack of IspC led to attenuated virulence in mice, evidenced by a significant reduction in bacterial counts in livers and brains and no mortality compared with the wild-type. Furthermore, the data from assays using various eukaryotic cells for adhesion, invasion, actin tail formation, plaque formation and intracellular growth indicated that the mutant was severely attenuated in virulence in a cell culture model in a cell type-dependent manner. The findings that (i) the mutant was impaired for adhesion to certain eukaryotic cells, and (ii) both purified IspC and epithelial cells and Vero cells, supported the role of IspC as an adhesin in virulence. The $\Delta$ ispC mutant exhibited a marked defect in adhesion to and invasion of SCP cells but not human brain microvascular endothelial cells (HBMEC), suggesting that IspC is necessary for crossing the blood-cerebrospinal fluid barrier. Proteomic and immunological analysis showed a reduced surface expression of some known or putative virulence factors (e.g. ActA, InIC2 and a flagellin homologue, FlaA) due to IspC deficiency. Altogether, this study demonstrates that IspC, expressed as a minor autolysin in vitro, is not important for cell division or separation but is essential for full virulence of $L$. monocytogenes in vivo.
\end{abstract} its C-terminal cell wall-binding domain were capable of binding sheep choroid plexus (SCP)
}

\section{INTRODUCTION}

Autolysins are peptidoglycan hydrolases that are capable of cleaving the covalent bonds in the cell wall peptidoglycan (murein) of bacteria from which they are produced (Shockman \& Holtje, 1994). Several autolysins from Listeria monocytogenes, a Gram-positive intracellular bacterium that infects humans leading to the development of a severe food-borne illness, including p60, P45, Ami, MurA and Auto, have been identified and characterized (Popowska, 2004). Bacterial autolysins are involved or implicated in pathogenesis (Berry et al., 1989; Cabanes et al., 2004; Canvin et al., 1995; Lenz et al., 2003; Lock et al., 1992; Mani et al., 1994; Milohanic et al., 2001) in addition to their biological functions, involving cell wall expansion,

Abbreviations: CWBD, cell wall-binding domain; GW module, glycinetryptophan dipeptide module; TEM, transmission electron microscopy. cell division, cell separation, chemotaxis, biofilm formation, genetic competence, protein secretion, antibioticinduced lysis, sporulation, and formation of flagella (Smith et al., 2000). Autolysin-deficient mutants, including an LytA mutant of Streptococcus pneumoniae (Berry \& Paton, 2000), an AtlE mutant of Staphylococcus epidermidis (Rupp et al., 1999), and Ami, Auto, p60 and MurA mutants of $L$. monocytogenes (Cabanes et al., 2004; Lenz et al., 2003; Milohanic et al., 2001; Pilgrim et al., 2003) are less virulent in animal models than their parental wild-type strains. The mechanisms that underlie the involvement of autolysins in virulence are not fully understood and need to be investigated further. The autolytic activity and the cell wall-binding domain (CWBD) of bacterial autolysins have been shown to be involved in virulence. LytA of Strep. pneumoniae and MurA and p60 of L. monocytogenes are thought to mediate the release of cytoplasmic toxins or 
proinflammatory degraded cell wall components resulting in subsequent tissue injuries (Jedrzejas, 2001; Lenz et al., 2003; Lock et al., 1992; Tuomanen, 2000). Restoration of virulence in an $L$. monocytogenes p60-defective mutant strain requires expression of the full-length autolysin p60 with an intact catalytic domain (Lenz et al., 2003). The CWBDs of autolysins, made up of repeated glycinetryptophan (GW) dipeptide modules, contribute to bacterial virulence by promoting adherence to eukaryotic cells, as shown for Ami of L. monocytogenes (Milohanic et al., 2001), and to extracellular matrix proteins, as shown for Aas of Staphylococcus saprophyticus (Hell et al., 1998) and AtlC of Staphylococcus caprae (Allignet et al., 2002).

We have recently demonstrated the peptidoglycan hydrolase (autolysin) activity of IspC, an L. monocytogenes (serotype $4 \mathrm{~b}$ ) immunogenic surface protein of 774 aa with a calculated molecular mass of $85.87 \mathrm{kDa}$ and a theoretical pI of 9.4 (Wang \& Lin, 2007; Yu et al., 2007). IspC is similar in modular domain structure to Auto and Ami of $L$. monocytogenes and contains an N-terminal catalytic domain (amino acids 24-197) and a C-terminal CWBD (amino acids 198-774) consisting of seven GW modules (Wang et al., 2007; Wang \& Lin, 2007). Several lines of experimental evidence accumulated in our recent studies (Wang \& Lin, 2007; Yu et al., 2007) have suggested that IspC is potentially involved in pathogenesis. This evidence includes the following: (i) antibody to IspC is present in rabbits infected with live L. monocytogenes but not in animals receiving heat-killed bacteria, suggesting that this protein is specifically induced or upregulated in vivo during infection; (ii) IspC is localized on the cell surface; and (iii) the protein possesses autolytic activity. Both Ami and Auto of $L$. monocytogenes are involved in pathogenesis, but they act via different mechanisms (Cabanes et al., 2004; Milohanic et al., 2001). Ami contributes to bacterial adhesion to eukaryotic cells, whereas Auto is not involved in adhesion but contributes to the entry of L. monocytogenes into eukaryotic cells. This suggests the necessity of examining the role of the newly identified $L$. monocytogenes autolysin IspC in pathogenesis.

Here we conducted an analysis of the L. monocytogenes is $p C$ gene and its product using genetic and proteomic approaches in an attempt to define the biological function of this cell-wall-anchored peptidoglycan hydrolase and its role in virulence. Data obtained from this investigation indicate that IspC is a novel factor that contributes to the virulence of $L$. monocytogenes through mechanisms involving the adhesive properties of the C-terminal CWBD, and that it regulates the surface display of other virulence factors, presumably by the autolytic activity of its $\mathrm{N}$ terminal catalytic domain.

\section{METHODS}

Bacterial culture and plasmid vector. The L. monocytogenes serotype 4b strain LI0521 (wild-type) and its ispC in-frame deletion mutant strain $(\Delta i s p C)$ were grown in brain heart infusion $(\mathrm{BHI})$ broth or on BHI agar plates. Escherichia coli DH5 $\alpha$ was used in cloning experiments and cultured in Luria-Bertani (LB) medium.

Purification of recombinant proteins. The recombinant IspC and its C-terminal CWBD (amino acids 198-774) fused C-terminally to a GFP variant optimized for brighter fluorescence when excited by UV light (GFPuv-CBD1) were expressed in E. coli and purified to electrophoretic homogeneity by using Ni-NTA agarose affinity chromatography followed by SP Sepharose cation-exchange chromatography (for IspC only), as described previously (Wang et al., 2007; Wang \& Lin, 2007).

Construction of ispC in-frame deletion mutant. A DNA fragment composed of the 453 bp sequence upstream of the ispC ORF and the first three codons was derived by PCR from the L. monocytogenes (serotype 4b strain LI0521) genomic DNA with the primer pair P517 (5'-ATCGAGCTCAGGAAAATCAAAAAGCTCCTCA-3', SacI site underlined) and P496 (5'-TAAAAGCTCGATTTATCATTATTCTCCAACCA-3'). A DNA fragment containing the 405 bp sequence downstream of the ispC ORF and the last eight C-terminal codons was similarly obtained by PCR with the primer pair P495 (5'AATGATAAATCGAGCTTTTACAAACGTTAAATAGAC-3') and P518 (5'-AAAGTCGACTGAGTGGAAAGGCTATGCTG-3', SalI site underlined). The two PCR fragments were spliced together as described previously (Wang \& Lin, 2007) using primers P495 and P496, resulting in an internal deletion of 764 aa of the coding sequence within ispC. The fused DNA fragment was inserted into the SacI and SalI sites of pAUL-A (kindly provided by Professor T. Chakraborty, Institute of Medical Microbiology, Justus-Liebig University, Germany) to create pAUL-A $\Delta$ ispC. Following electroporation of the recombinant plasmid into competent $L$. monocytogenes that had been prepared as described elsewhere (Park \& Stewart, 1990), bacteria were screened for targeted gene deletion as described elsewhere (Schaferkordt et al., 1998). A $\Delta$ isp C mutant was confirmed by PCR analysis of the genomic DNA using a primer pair internal to the deletion region [P304 (5'-GATGGTAAAGTCATTGGCTG-3') and P285 (5'-TTAGCCTCGCGTATCAACTG-3')] and a primer pair external to the deletion region [P481 (5'-CAGCACAAGAAGTTGCTCAAAA-3') and P518], and by sequencing the PCR products.

Confirmation of the $\Delta i s p C$ mutant at the protein level. Surface expression of IspC was examined by immunofluorescence microscopy analysis of live bacteria (the $\triangle i s p C$ mutant and the wild-type) probed with rabbit antiserum raised against purified recombinant IspC ( $\mathrm{R} \alpha \mathrm{IspC}$ ), as described previously (Wang \& Lin, 2007). IspC in the total protein extracts (see below) from the mutant and wild-type strains was also analysed by Western blotting using R $\alpha$ IspC. The bacteriolytic activity of the wild-type and the $\Delta i s p C$ mutant was assessed by analysis of the cell surface protein extracts (see below) equivalent to $1 \mathrm{ml}$ of culture with an $\mathrm{OD}_{620}$ of 1.0 in a $12 \%$ renaturing SDS-PAGE gel containing $0.2 \% \quad(\mathrm{w} / \mathrm{v})$ autoclaved Micrococcus lysodeikticus ATCC 4698 (Sigma), as described previously (Wang \& Lin, 2007).

Passage of $\boldsymbol{L}$. monocytogenes in mice. The $\Delta i s p C$ mutant and wild-type strains were passaged in vivo in mice to eliminate the possibility that in vitro growth may reduce the bacterial virulence.

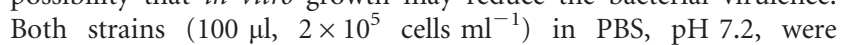
intravenously inoculated into BALB/c mice. Each strain recovered from livers of infected mice at day 3 post-infection was grown to $\mathrm{OD}_{620} 0.8$, washed with $\mathrm{PBS}$, and stored in $1 \mathrm{ml}$ aliquots at appropriate concentrations in PBS containing $15 \%(\mathrm{v} / \mathrm{v})$ glycerol at $-80{ }^{\circ} \mathrm{C}$ until use.

Phenotypic analysis. Comparative phenotypic analysis of the $\Delta i s p C$ mutant and the wild-type with respect to their in vitro growth, and biochemical and morphological characteristics was performed. The 
growth curve was established by subculturing each strain at the same starting concentration from the overnight culture in triplicates in $50 \mathrm{ml}$ BHI broth ( $\mathrm{pH} 7.2$ ), BHI broth ( $\mathrm{pH} 7.2$ ) containing $0.3 \mathrm{M}$ $\mathrm{NaCl}$ (osmotic stress), and BHI broth ( $\mathrm{pH} 4.5$; acidic stress). Samples of cultures were taken at various time points for $48 \mathrm{~h}$ to measure $\mathrm{OD}_{620}$. The catalase test, Christie-Atkins-Munch-Petersen (CAMP) test (Staphylococcus aureus and Rhodococcus equi), $\mathrm{H}_{2} \mathrm{~S}$ test, oxidase test, nitrate reduction test and motility test $\left(25\right.$ and $\left.35{ }^{\circ} \mathrm{C}\right)$ were conducted according to established methods (MacFaddin, 2000). Carbohydrate utilization under fermentative and oxidative conditions was tested by using API $50 \mathrm{CH}$ strips (bioMérieux) using $\mathrm{CHB} / \mathrm{E}$ medium according to the manufacturer's instructions. The colony morphology of the mutant was examined by culturing bacteria on tryptic soy sheep blood agar (TSBA) plates. The microscopic morphology of bacterial cells was examined at various phases of growth (i.e. early exponential, mid-exponential, late-exponential and stationary phases) using phase-contrast light microscopy, transmission election microscopy (TEM) and cross-sectional TEM.

Extraction of total bacterial proteins. The $\Delta i s p C$ mutant and wildtype strains at early exponential growth phase, equivalent to $5 \mathrm{ml}$ of culture with an $\mathrm{OD}_{620}$ of 1.0 , were treated with chloramphenicol $\left(20 \mu \mathrm{g} \mathrm{ml}^{-1}\right)$ to inhibit protein synthesis and washed with ice-cold PBS containing proteinase inhibitor cocktail (Roche) and $20 \mu \mathrm{g}$ chloramphenicol ml ${ }^{-1}$ (PBS-CC). Bacteria were lysed in $500 \mu \mathrm{l}$ PBS$\mathrm{CC}$ in a FastProtein Blue tube on a FastPrep apparatus FP120 (Qbiogene) with five bursts of $45 \mathrm{~s}$ on setting 6.5. The cell lysates were boiled for $10 \mathrm{~min}$ after addition of $500 \mu \mathrm{l} 2 \times$ SDS-PAGE sample buffer [0.1 M Tris/HCl, pH 6.8, $40 \%(\mathrm{v} / \mathrm{v})$ glycerol, $20 \%(\mathrm{v} / \mathrm{v}) \beta$ mercaptoethanol, $4 \%(\mathrm{w} / \mathrm{v})$ SDS and $0.02 \%(\mathrm{w} / \mathrm{v})$ bromophenol blue] and centrifuged at $14100 \mathrm{~g}$ to collect total bacterial proteins (the supernatant) for SDS-PAGE analysis.

Extraction of bacterial surface proteins. Samples of the $\Delta i s p C$ mutant and wild-type strains equivalent to $50 \mathrm{ml}$ of culture with an $\mathrm{OD}_{620}$ of 0.5 were collected at various growth phases (early exponential, mid-exponential, late-exponential and stationary) and washed as described above. Cell pellets were resuspended in $1 \mathrm{ml} 2 \times$ SDS-PAGE sample buffer and boiled for $10 \mathrm{~min}$. The supernatants containing surface-extracted proteins were collected by centrifugation at $14100 \mathrm{~g}$ for $5 \mathrm{~min}$ and stored at $-20{ }^{\circ} \mathrm{C}$ until use.

Analysis of cell surface proteins by MS and Western blotting. Bacterial surface proteins $(150 \mu \mathrm{l})$ from the $\Delta i s p C$ mutant and wildtype strains were separated by SDS-PAGE using a $4 \%$ stacking gel and a $12 \%$ resolving gel on a Protein II xi system (Bio-Rad) and stained with Coomassie brilliant blue. Resolved protein bands of interest were excised from the wild-type samples and subjected to in-gel tryptic digestion for liquid chromatography-tandem MS (LC-MS-MS) analysis, as described elsewhere (Vasilescu et al., 2005). The MS-MS data were then analysed against the protein database using the Mascot search engine. Western blot analysis of surface proteins was performed as described previously (Wang \& Lin, 2007), with rabbit anti-InlA, anti-InlB, anti-ActA and antiInlC2 antibodies.

In vivo virulence assay. Six- to eight-week-old female $\mathrm{BALB} / \mathrm{c}$ mice were used in in vivo infection experiments with the $\Delta i s p C$ mutant and the wild-type strain. For each bacterial strain, four groups of six mice each were intravenously inoculated with $5 \times 10^{4}$ bacteria in $100 \mu \mathrm{l}$ PBS. Brains, livers and spleens were aseptically removed from one particular group $(n=6)$ at $6,24,48$ and $72 \mathrm{~h}$ post-infection, homogenized in $5 \mathrm{ml} 1 \%$ buffered peptone water (BPW) using a stomacher, and spread on BHI agar plates with $0.1 \mathrm{ml}$ of homogenates at 10 -fold serial dilutions. Bacteria were enumerated after incubation at $37{ }^{\circ} \mathrm{C}$ for $24 \mathrm{~h}$.
Cell lines and cell culture. The cell lines Caco-2 (human colon carcinoma enterocyte-like epithelial cell), Hep-G2 (human hepatocellular carcinoma cell), Vero (African green monkey kidney cell), L132 (human embryonic lung fibroblast), Hela (human cervical epithelial cell), HBMEC (human brain microvascular endothelial cell), SCP (sheep choroid plexus epithelial cell), L2 (murine fibroblast cell) and J774 (murine macrophage) were grown in MEM complete medium $(1 \times$ minimal essential medium supplemented with $10 \%$ heatinactivated fetal bovine serum (FBS) or $5 \%$ horse serum for SCP, $0.1 \mathrm{mM}$ non-essential amino acids, $2 \mathrm{mM}$ L-glutamine and $0.15 \%$ sodium bicarbonate). Sodium pyruvate was added at $1 \mathrm{mM}$ into the MEM complete medium for Hep-G2. HBMEC (Cell Systems) was cultured on gelatin-coated surface in Cell Systems complete (CS-C) medium according to the manufacturer's instructions. All eukaryotic cells were grown at $37{ }^{\circ} \mathrm{C}$ and $5 \% \mathrm{CO}_{2}$ in a humid $\mathrm{CO}_{2}$ incubator.

Adhesion, invasion and intracellular growth assays. The ability of bacteria with respect to adhesion to-, invasion of-, and intracellular growth within eukaryotic cells was assessed basically as described elsewhere (Bergmann et al., 2002; Rowan et al., 2000), with some minor modifications. Briefly, $2 \times 10^{5}$ cells $\left(1 \times 10^{5} \mathrm{HBMEC}\right.$ cells $)$ were seeded in 24-well tissue culture plates (VWR), grown for $48 \mathrm{~h}$, and infected with $0.5 \mathrm{ml}$ bacterial suspension $(\triangle i s p C$ mutant or wild-type strain) in MEM complete medium (for HBMEC, $25 \mathrm{mM}$ HEPES and $1 \mathrm{mM}$ sodium pyruvate was added to the medium) for $1 \mathrm{~h}$ at the desired m.o.i. for each cell line: m.o.i. 50 for Hela and L132, m.o.i. 100 for Vero, m.o.i. 20 for Caco-2, Hep-G2, SCP and HBMEC, and $2 \times 10^{5}$ bacteria for J774. For the adhesion assay, eukaryotic cells were washed five times with $3 \mathrm{ml}$ PBS [Dulbecco's Modified Eagle Medium (DMEM, high glucose) for HBMEC] and lysed with $1 \mathrm{ml} 1 \%(\mathrm{v} / \mathrm{v})$ Triton X-100. Viable bacteria were enumerated by plating $0.1 \mathrm{ml}$ lysates at 10-fold serial dilutions on BHI agar plates. For the invasion assay, infected eukaryotic cells were washed and incubated in $1 \mathrm{ml}$ medium containing $100 \mu \mathrm{g}$ gentamicin $\mathrm{ml}^{-1}$ for $1.5 \mathrm{~h}$. After washing, surviving intracellular bacteria were quantified as described above. For intracellular growth assays, infected eukaryotic cells were washed and incubated in medium containing $100 \mu \mathrm{g}$ gentamicin $\mathrm{ml}^{-1}$. Surviving bacteria were quantified as above at 2, 4, 6 and $8 \mathrm{~h}$ after addition of gentamicin. All cellular adhesion, invasion and growth assays were performed in triplicate.

Actin tail formation. J774 cells $\left(2 \times 10^{5}\right.$ per well $)$ were seeded into two-well Lab-TekII chamber slides (Fisher) and grown to $\sim 90 \%$ confluence. After washing with DMEM minus FBS $\left(37^{\circ} \mathrm{C}\right)$, cells were infected with $10^{7}$ bacteria (mutant or wild type) in $1 \mathrm{ml}$ of the same medium for $1 \mathrm{~h}$, and then incubated in complete MEM containing $100 \mu \mathrm{g}$ gentamicin $\mathrm{ml}^{-1}$ for an additional 3 or $6 \mathrm{~h}$. After washing with PBS, cells were fixed in $4 \%(\mathrm{w} / \mathrm{v})$ paraformaldehyde in PBS for $12 \mathrm{~min}$, permeabilized in $0.2 \%(\mathrm{v} / \mathrm{v})$ Triton X-100 in PBS for $8 \mathrm{~min}$, and blocked in $3 \%(\mathrm{w} / \mathrm{v})$ BSA in PBS for $30 \mathrm{~min}$. Bacteria were stained with rabbit antiserum to ActA for $1 \mathrm{~h}$ followed by incubation with Alexa Fluor 647 goat anti-rabbit secondary antibody (Invitrogen) for $1 \mathrm{~h}$. Actin tails were stained with Alexa Fluor 488 Phalloidin (Invitrogen) for $30 \mathrm{~min}$. Nuclei were stained with $4^{\prime}, 6$ diamidino-2-phenylindole (DAPI; Sigma) at $10 \mu \mathrm{M}$ in PBS for $3 \mathrm{~min}$. Slides were mounted with Vectashield mounting medium (Vector Laboratories) and examined on an Olympus BX51 fluorescence microscope (Olympus Canada).

Immunofluorescence and immunogold TEM. Immunofluorescence and immunogold TEM procedures (Wang \& Lin, 2007) were used to detect the in situ surface expression of IspC or ActA in live bacteria of the $\Delta i s p C$ mutant and wild-type strains.

Plaque assay. Murine L2 cells $\left(3 \times 10^{5}\right.$ per well $)$ were seeded into six-well plates (VWR) and grown to confluence. After washing with DMEM minus FBS $\left(37^{\circ} \mathrm{C}\right)$, cells were infected with $10^{6}$ bacteria 
( $\triangle i s p C$ mutant or wild-type strain) in $1 \mathrm{ml}$ of the same medium for $1 \mathrm{~h}$. After washing with complete MEM medium $\left(37^{\circ} \mathrm{C}\right)$ containing $10 \mu$ gentamicin $\mathrm{ml}^{-1}, 3 \mathrm{ml}$ agar overlay $\left(42^{\circ} \mathrm{C}\right)$ containing $1 \times$ DMEM, $10 \%$ FBS, $10 \mu$ gentamicin $\mathrm{ml}^{-1}$ and $0.7 \%$ agarose was added to each well, and the plates were incubated for 4 days at $37^{\circ} \mathrm{C}$. Plaques were visualized on a Zeiss Axiovert-10 inverted microscope equipped with a Nikon digital camera at a total magnification of 45 and their relative sizes (diameters) estimated using ImageJ version 1.37a (National Institutes of Health).

Cell binding assay. Eukaryotic cells $\left(1.7 \times 10^{5}\right.$ for SCP, $1 \times 10^{5}$ for Vero) were seeded into two- or four-well chamber slides, grown to $\sim 70 \%$ confluence, and fixed as above. Fixation followed by permeabilization treatment was used as a control for surface binding. Cells were blocked in 3\% BSA in PBS for $30 \mathrm{~min}$ and incubated with either IspC $\left(3.5 \mu \mathrm{g} \mathrm{ml}^{-1}\right)$ or GFPuv-CBD1 $(1.14 \mu \mathrm{M})$ for $2.5 \mathrm{~h}$. For IspC binding only, cells were further incubated with R $\alpha$ IspC at $1: 250$ in $3 \% \mathrm{BSA}$ in PBS for $1 \mathrm{~h}$ followed by interaction with FITCconjugated goat anti-rabbit IgG (Zymed) at 1:50 for $30 \mathrm{~min}$. Slides were processed and examined using a fluorescence microscope as described above. Preimmune serum and purified GFPuv were used as negative controls.

Statistical analysis. The data from in vivo virulence, adhesion, invasion, intracellular growth and plaque assays with the $\Delta i s p C$ mutant and the wild-type strain were statistically analysed using Student's $t$ test, and $P$ values $<0.05$ were considered statistically significant.

\section{RESULTS}

\section{Construction and phenotypic characterization of an ispC in-frame deletion mutant}

To elucidate the biological function of the $86 \mathrm{kDa}$ autolysin IspC (Wang \& Lin, 2007) and its potential role in virulence, an IspC-deficient L. monocytogenes strain was successfully created from the parental strain L. monocytogenes serotype $4 \mathrm{~b}$ via homologous recombination using the pAUL-A-based integration-excision technique. This mutant, which has a chromosomal in-frame deletion of 2292 bp within the 2325 bp ispC ORF (Fig. 1a), designated $\triangle i s p C$, was confirmed by PCR with two sets of primer pairs (Fig. 1b) followed by DNA sequencing. With primers P304 and P285, which target the deleted region, no PCR product was obtained from the $\Delta i s p C$ mutant chromosomal DNA, in contrast to a product derived from the wild-type strain that corresponded to its calculated size of $452 \mathrm{bp}$. With primers P481 and P518, which anneal outside the deleted region, a PCR product close to the expected size of $993 \mathrm{bp}$ was amplified from the mutant chromosomal DNA and, as expected, was smaller than that $(\sim 3200 \mathrm{bp})$ derived from the wild-type. DNA sequencing of the PCR product derived from the $\triangle i s p C$ mutant with primers P481 and P518 also confirmed a target deletion of 2292 bp within the ispC ORF.

The $\Delta i s p C$ mutant exhibited the abrogation of IspC expression in in vitro culture, as evidenced by the data from three separate experiments. (i) Western blot analysis of total bacterial proteins revealed that a protein band of $\sim 86 \mathrm{kDa}$ from the wild-type recognized by rabbit anti-IspC polyclonal antibody (R $\alpha \mathrm{IspC})$ was not present in the mutant (Fig. 1c). (ii) Renaturing SDS-PAGE analysis of the surface protein extract for peptidoglycan hydrolase activity with respect to $M$. lysodeikticus cell wall showed that a bacteriolytic band of $\sim 86 \mathrm{kDa}$ associated with the wild type was not found in the mutant (Fig. 1d). (iii) Immunofluorescence microscopy with R $\alpha I s p C$ showed no fluorescent staining on live mutant bacteria but detected IspC on the surface of wild-type bacteria (Fig. 1e). Interestingly, surface peptidoglycan hydrolase activity profiles from the wild-type and the $\Delta i s p C$ mutant showed that IspC is a minor autolysin in in vitro culture.

The $\Delta i s p C$ mutant was indistinguishable from the wildtype strain with respect to the following: colony morphology on TSBA plates, bacterial morphology at various growth phases in BHI broth at $37{ }^{\circ} \mathrm{C}$ as revealed by phasecontrast microscopy (Fig. 1f), TEM (data not shown) and sectional TEM (Fig. 1g), motility at 25 and $35{ }^{\circ} \mathrm{C}$, haemolysis on sheep blood agar plates, carbohydrate utilization on API $50 \mathrm{CH}$ strips, growth curve at $37^{\circ} \mathrm{C}$ in BHI broth or growth in BHI under acidic ( $\mathrm{pH} 4.5)$ or osmotic $(0.3 \mathrm{M} \mathrm{NaCl})$ stress, and a number of biochemical tests [i.e. catalase test, CAMP test (Staph. aureus and $R$. equi), $\mathrm{H}_{2} \mathrm{~S}$ test, oxidase test and nitrate reduction test].

\section{IspC is required for virulence in vivo}

To evaluate whether the is $p C$ gene contributes to virulence, $\mathrm{BALB} / \mathrm{c}$ mice were infected intravenously with the $\Delta i s p C$ mutant and the wild-type strain. The deficiency of IspC in bacteria significantly $(P<0.01)$ reduced the bacterial load in the target organs (by approximately fivefold in brain and approximately sevenfold in liver) at $48 \mathrm{~h}$ post-infection with the exception of spleen, while the bacterial counts in these organs were similar between the mutant and the wildtype at 6 and $24 \mathrm{~h}$ (Fig. 2). The requirement of IspC for virulence was further demonstrated by the fact that the mutant did not cause any mortality at $72 \mathrm{~h}$, in contrast to the death of two out of six mice infected with the wild-type strain. This result prompted us to further investigate the role of IspC in virulence at the various stages of the infectious cycle, using cultured eukaryotic cells.

\section{Involvement of IspC in bacterial adhesion in a cell type-dependent manner}

In earlier studies, GW modules of several autolysins, including Ami of L. monocytogenes (Milohanic et al., 2001), Aas of Staph. saprophyticus (Hell et al., 1998) and AtlC of Staph. caprae (Allignet et al., 2002), were shown to be involved in bacterial adhesion. To determine if the surface autolysin IspC, which contains seven GW modules in its Cterminal CWBD (Wang \& Lin, 2007) promotes the adherence of bacteria to eukaryotic cells, the $\Delta i s p C$ mutant and the wild-type were assayed for binding to several types of eukaryotic cells (Fig. 3). The primary attachment of the $\Delta$ ispC mutant to Hep-G2, Vero and SCP was reduced by 
(a)

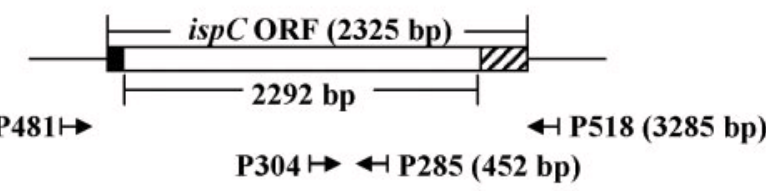

(b)

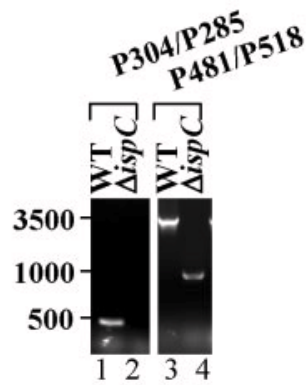

(c) 107

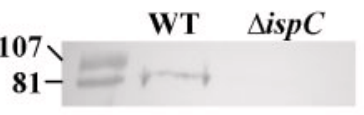

(e)
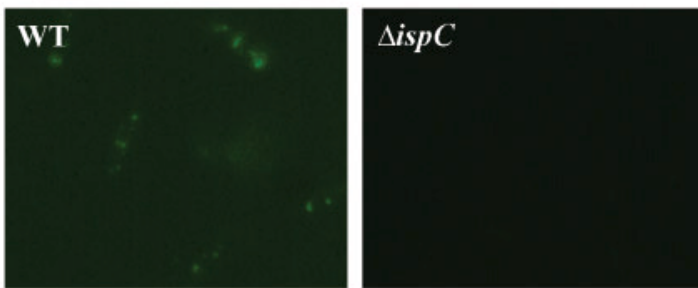

(f)

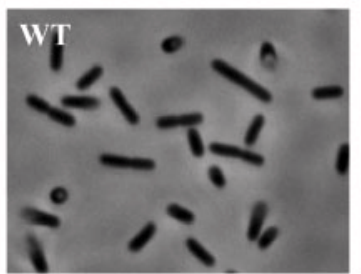

(d)
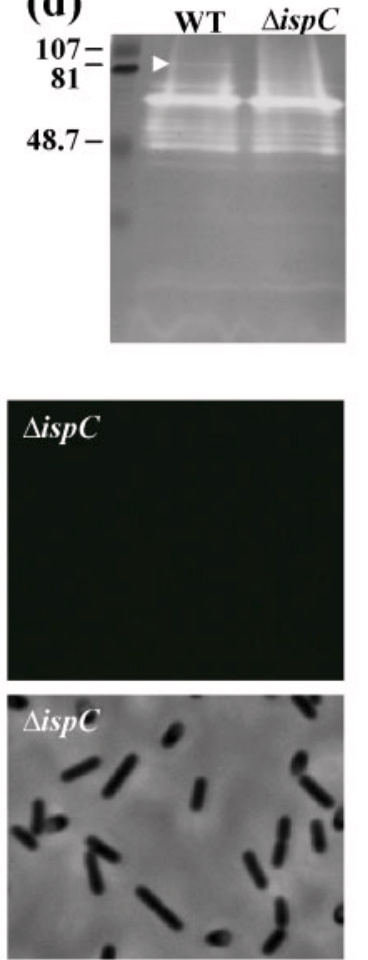

(g)

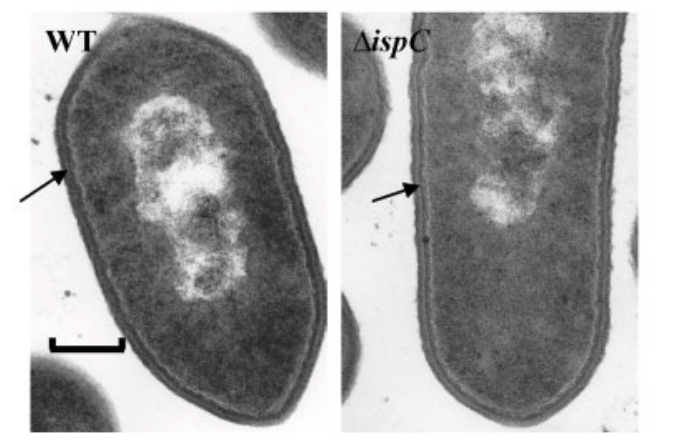

approximately two- to threefold $(P<0.01)$ in comparison to that of the wild-type strain, while both strains were similar in adhesion to Caco-2, L132, Hela, HBMEC and mouse macrophage J774. This indicates that IspC plays a role in mediating bacterial adhesion to normally nonphagocytic cells in a cell type-dependent manner.

\section{IspC is required for invasion in a cell type-dependent manner}

To determine whether IspC contributes to the invading capability of $L$. monocytogenes, internalization of the $\Delta i s p C$
Fig. 1. Comparison of a $\triangle i s p C$ mutant strain of $L$. monocytogenes with the wild-type at DNA, protein and microscopic levels. (a) Schematic representation of the is $p C$ gene locus of the wild-type strain and the corresponding gene deletion in the mutant. The annealing positions of the primer pairs P304/P285 and P481/ P518 used to confirm the $\triangle i s p C$ mutant are indicated by arrows. The numbers in parentheses indicate the predicted sizes of PCR products amplified from the wild-type genomic DNA using the corresponding primers. The deleted sequence coding for amino acids 4-767 of IspC, the first three codons and the last eight codons of IspC are depicted by an open bar, a filled bar and a hatched bar, respectively. (b) Gel electrophoresis analysis of PCR products derived from the chromosomal DNAs of the mutant and the wild-type (WT). Lanes: 1, product from the wild-type with primer pair P304/P285; 2, product from the $\Delta i s p C$ mutant with primer pair P304/P285; 3, product from the wild-type with primer pair P481/P518; 4, product from the $\triangle i s p C$ mutant with primer pair P481/P518. (c) Western blot analysis of the total proteins extracted from the mutant and wild-type (WT) strains. Each lane containing the proteins from bacterial cells equivalent to $0.1 \mathrm{ml}$ culture with an $\mathrm{OD}_{620}$ of 1.0 was probed with rabbit anti-lspC antiserum ( $\mathrm{R} \alpha \mathrm{lspC}$ ). The molecular masses of protein standards (in $\mathrm{kDa}$ ) are shown on the left. (d) The bacteriolytic profiles of the surface proteins from the mutant and wild-type (WT) strains. The bacteriolytic activity was analysed in a renaturing SDS-PAGE $(12 \%)$ gel containing $0.2 \%(w / v)$ M. lysodeikticus. Each lane contains the surface proteins extracted from bacterial cells equivalent to $1 \mathrm{ml}$ culture with an $\mathrm{OD}_{620}$ of 0.5 . The bacteriolytic band of $\sim 86 \mathrm{kDa}$ corresponding to the IspC protein is indicated by an arrow. (e) Detection of the surface expression of IspC in the mutant and wild-type (WT) strains by immunofluorescence staining with RalspC. (f) Phase-contrast micrographs of the mutant and the wild-type harvested at the mid-exponential growth phase. (g) Sectional TEM micrographs of the mutant and wild-type (WT) strains at mid-exponential growth phase. The bacterial cell walls are indicated by arrows. Magnification, $\times 50000 ;$ bar, $0.16 \mu \mathrm{m}$.

mutant and the wild-type into various normally nonphagocytic eukaryotic cells was investigated (Fig. 4). The mutant exhibited a reduction in internalization into Caco2, Hep-G2, Vero, L132 and SCP by two- to threefold $(P<0.01)$ relative to the wild-type, while it entered Hela and HBMEC as efficiently as the wild-type. Thus, IspC is involved in invasion of normally non-phagocytic cells by $L$. monocytogenes in a cell type-dependent manner.

\section{Direct binding of recombinant IspC and its C- terminal CWBD to the surface of SCP and Vero cells}

The observation that the $\Delta i s p C$ mutant possessed weaker adhesive and invasive characteristics with respect to certain eukaryotic cell types prompted us to further study the adhesive properties of purified IspC and its C-terminal CWBD (amino acids 198-774) fused to GFPuv (GFPuvCBD1) with respect to SCP and Vero cells; in these studies the mutant exhibited an impaired adhesion and invasion ability. Fluorescence microscopy showed that both the purified recombinant IspC and GFPuv-CBD1 bound to 

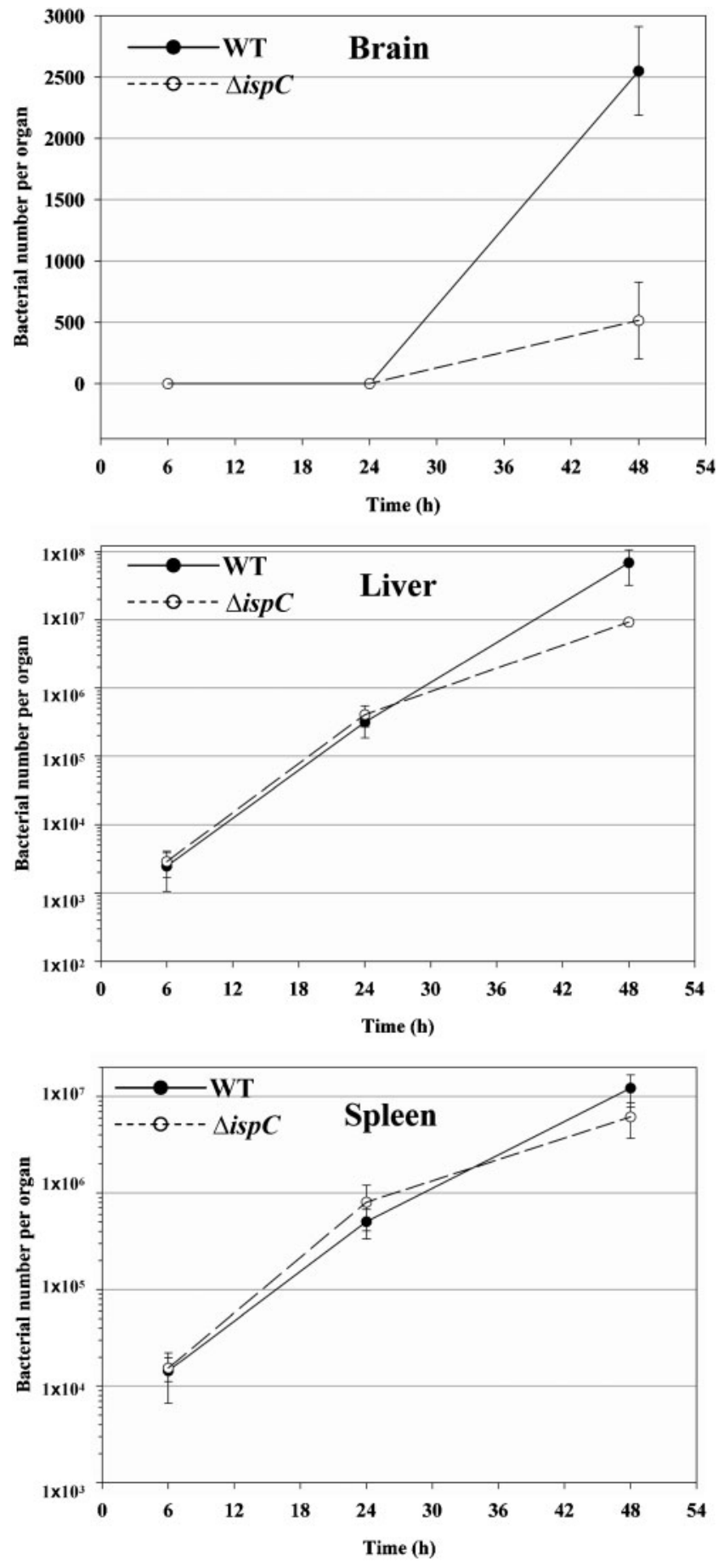

Fig. 2. Bacterial counts in the target organs of mice infected with the $\Delta i s p C$ mutant and the wild-type (WT) strain. Groups of BALB/ $c$ mice (six mice per group) were intravenously inoculated with $5 \times 10^{4}$ bacteria of each strain. The number of viable bacteria in brains, livers and spleens obtained at 6,24 and $48 \mathrm{~h}$ postinfection are presented as the mean $\pm \mathrm{SD}(n=6)$. Bacterial organ counts were not available at $72 \mathrm{~h}$ because two out of six mice infected with the wild-type strain died.
SCP and Vero (Fig. 5). Stronger fluorescent signals were observed for binding of these proteins to SCP than to Vero. Fixation and fixation followed by permeabilization of these eukaryotic cells prior to fluorescence staining resulted in similar fluorescence images of cells (data not shown), indicating that the binding event occurred on the cell surface. Binding of IspC or its C-terminal CWBD to the cell surfaces of SCP and Vero was specific, as no fluorescence staining on the cell surfaces was observed when cells were probed with rabbit preimmune serum or purified GFPuv. Under the same assay conditions, almost no fluorescence was observed on the surface of HBMEC probed with purified IspC or GFPuv-CBD1 (data not shown).

\section{Deletion of ispC impairs the display of surface proteins}

To determine whether the chromosomal deletion of is $p C$ would alter the display of bacterial surface proteins, including known virulence factors, as an alternative mechanism leading to the reduced adhesive and invasive capacity of the mutant, surface proteins from the $\Delta i s p C$ mutant and the wild-type at various growth phases were analysed by SDS-PAGE followed by MS (Fig. 6a) or by Western blotting (Fig. 6b) using specific antibodies. Two protein bands of $\sim 90$ and $\sim 30 \mathrm{kDa}$ present in the wild-type were undetectable in the mutant by SDS-PAGE, and were subsequently identified by MS as ActA and a flagellin protein (a FlaA homologue), respectively. Western blot analysis using rabbit antiserum to ActA showed that expression of ActA was not abolished in the mutant but was dramatically reduced at all growth phases, in contrast to the wild-type (Fig. 6b). The decrease in the amount of ActA was also observed with Western blot analysis of total cell lysates of the mutant (data not shown). Reduced surface display of ActA in the mutant was further demonstrated by immunofluorescence microscopy (Fig. $6 c$, top and middle panels) and immunogold TEM (Fig. 6c, bottom panel) probed with rabbit anti-ActA antibody. Western blot analysis using rabbit anti-InlC2 antibody revealed a significant reduction in InlC2 expression in the mutant, although this protein was not visible on the SDSPAGE gel. Three surface proteins having a similar expression level in the mutant and the wild-type were identified to be InlB precursor and p60, by MS, and InlA, by Western blot analysis using rabbit anti-InlA antibody. The absence of an alteration in InlB expression was further confirmed by Western blot analysis using rabbit anti-InlB antibody.

\section{IspC is involved in actin tail formation in the early stages of intracellular infection}

The observation that the surface display of a few proteins, including ActA, was strikingly reduced in the $\Delta i s p C$ mutant prompted us to further investigate whether the abrogation of IspC expression affects the intracellular motility of 


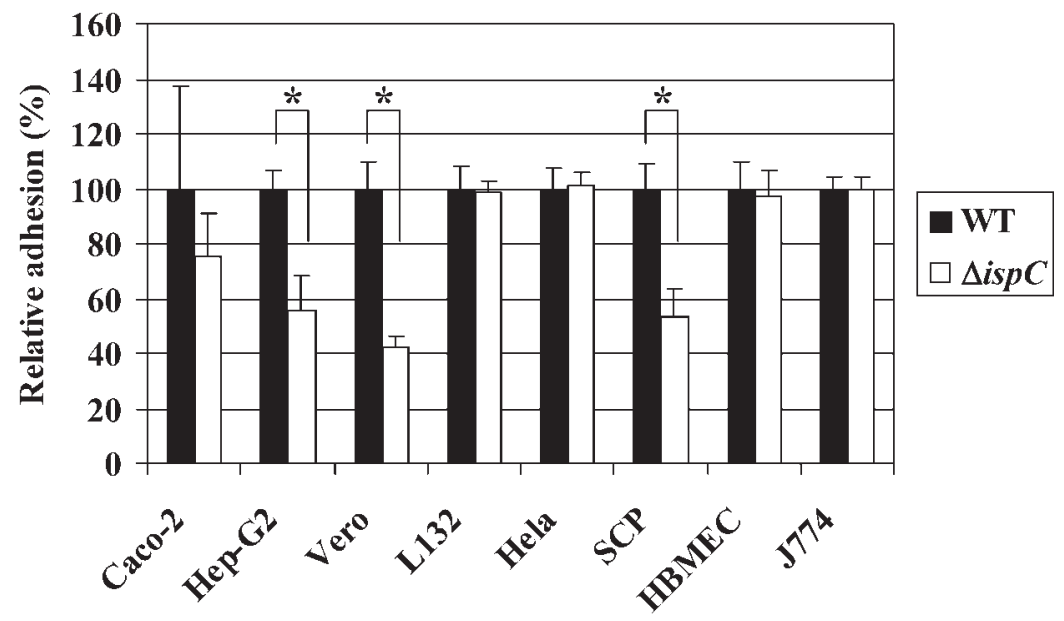

Fig. 3. Quantitative analysis of adhesion of the $\Delta i s p C$ mutant to various eukaryotic cell lines in comparison with the wild-type (WT). The mutant and wild-type strains were used to infect a particular cell line at a predetermined m.o.i. (see Methods) for $1 \mathrm{~h}$ and the bacteria associated with the eukaryotic cells were quantified by plating the cell lysates after washing away free bacteria. The quantity of mutant bacteria is calculated relative to that of the wild type (set as $100 \%$ ) and presented as the mean \pm SD $(n=3)$. * Statistically significant difference $(P<0.05)$.

bacteria by examining, in comparison with the wild-type, the formation of actin tails in the intracellular niche after infection with the mutant of mouse macrophage J774 cells. The mutation led to formation of much shorter and weaker actin tails at the early infection stage (i.e. $3 \mathrm{~h}$ ) than the wild-type, while at the late infection stage (i.e. $6 \mathrm{~h}$ ), formation of the actin tails was similar in the mutant and the wild-type (Fig. 7).

\section{IspC contributes to cell-to-cell spread}

A contribution of IspC to cell-to-cell spread of bacteria may be expected, as the formation of actin tails was impaired in early stages of infection with the $\Delta i s p C$ mutant. This was macroscopically confirmed by a plaque assay of a murine L2 fibroblast monolayer infected with the mutant as compared with the wild-type. The relative plaque size

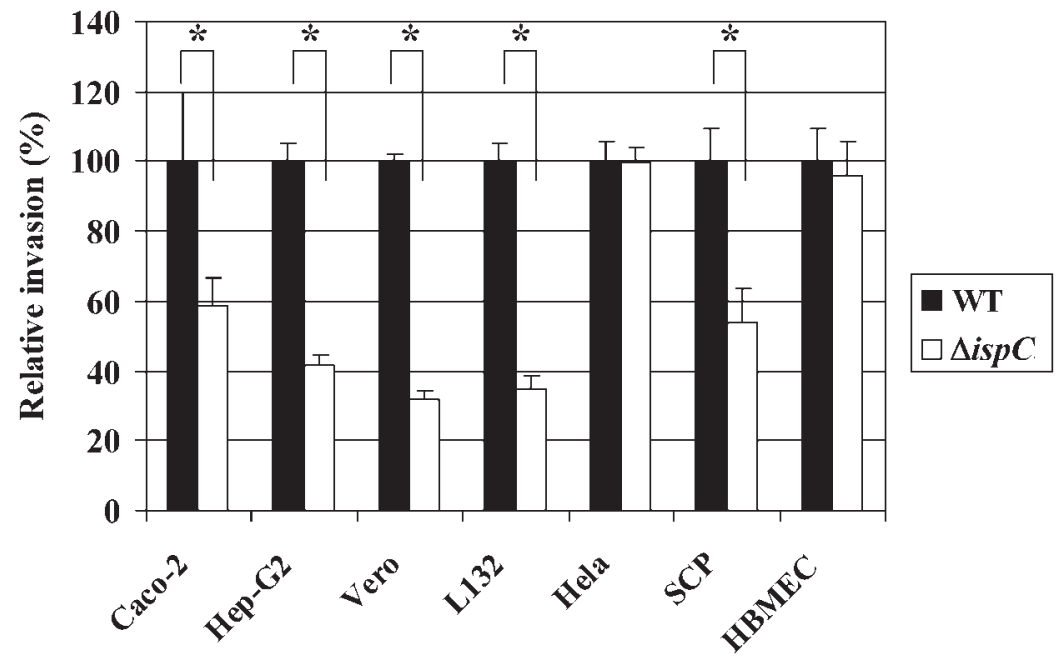

Fig. 4. Quantitative analysis of entry of the $\Delta i s p C$ mutant into various eukaryotic cell lines. The mutant and wild-type (WT) strains were used to infect a particular cell line in triplicate at a predetermined m.o.i. (see Methods) for $1 \mathrm{~h}$ followed by further incubation with $100 \mu \mathrm{g}$ gentamicin $\mathrm{ml}^{-1}$ for $1.5 \mathrm{~h}$. The intracellular bacteria were determined as in Fig. 3. The quantity of mutant bacteria is calculated relative to that of the wild type (set as $100 \%)$ and presented as the mean \pm SD $(n=3)$. * $\operatorname{Statistically}$ significant difference $(P<0.05)$. 

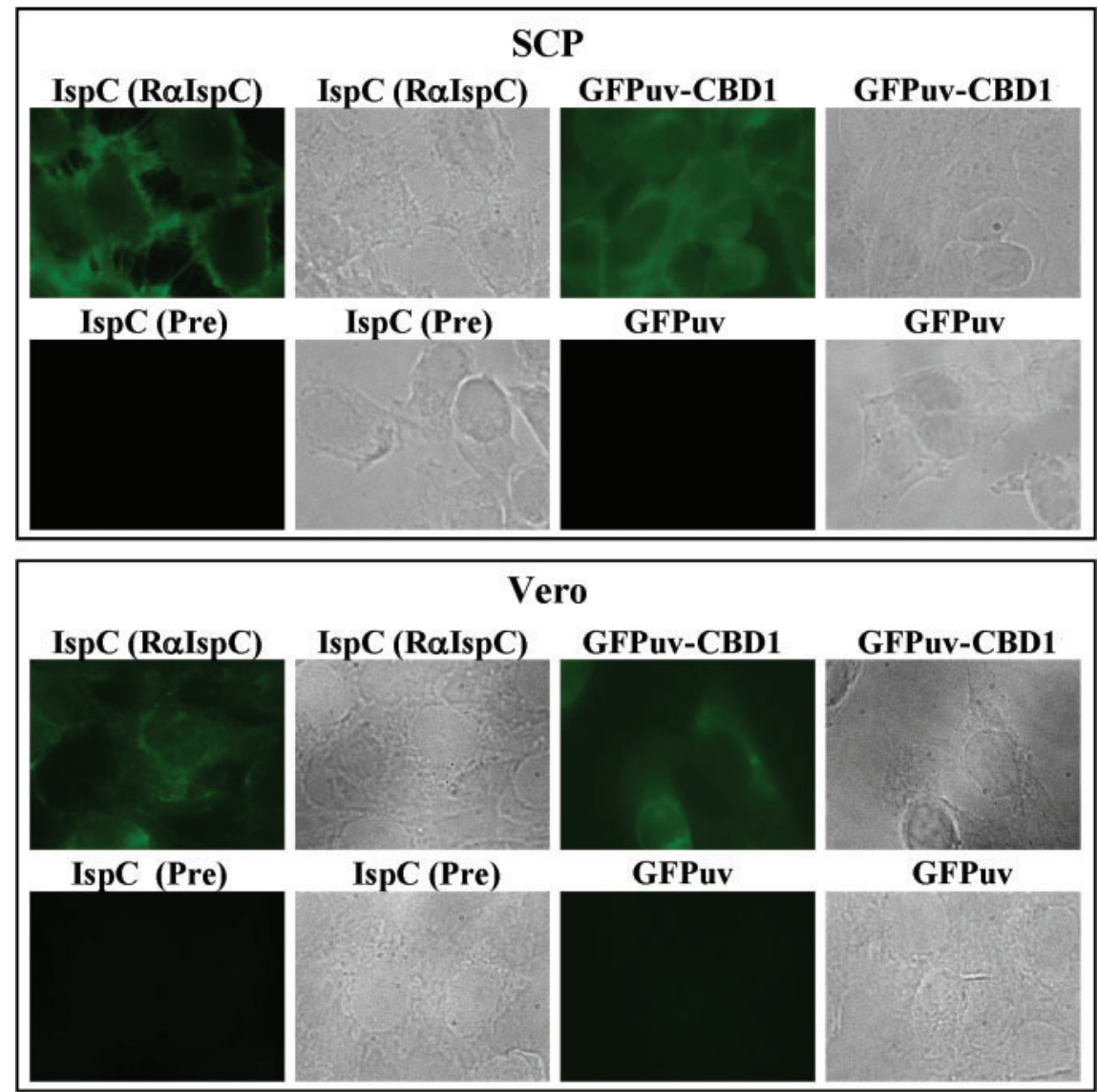

Fig. 5. Binding to SCP and Vero cells of purified recombinant IspC and an IspC CWBD (amino acids 198-774) fused with GFPuv (GFPuv-CBD1). Paraformaldehyde-fixed SCP and Vero cells were incubated with purified recombinant IspC (3.5 $\mu \mathrm{g}$ $\mathrm{ml}^{-1}$ ) or GFPuv-CBD1 $(1.14 \mu \mathrm{M})$. The bound IspC was detected by interaction with R $\alpha$ lspC followed by staining with FITCconjugated goat anti-rabbit lgG. The bound GFPuv-CBD1 was detected with the intrinsic fluorescence of the fusion protein. The rabbit preimmune serum (Pre) or GFPuv was used as a negative control. Fluorescence and phase-contrast images of eukaryotic cells to which IspC or GFPuv-CBD1 bound were viewed using a fluorescence microscope.

(expressed in arbitrary units; a.u.) formed by the mutant strain (175.5 \pm 18.444 a.u., $n=22)$ was significantly smaller $(P<0.01)$ than that of the wild-type $(206.375 \pm 25.595$ a.u., $n=24$ ) (Fig. 8), indicating the reduced capacity for bacterial cell-to-cell spread of the $\Delta i s p C$ strain.

\section{IspC contributes to bacterial intracellular growth at the later infection stage}

Autolysins have dual effects on bacterial growth and survival (favouring growth and causing bacterial death). The effect of IspC on intracellular survival and growth was assessed in two representative cell lines (Vero and J774) following infection with the $\triangle i s p C$ mutant and the wildtype over an $8 \mathrm{~h}$ time-course. The mutant showed an approximately twofold reduction in growth within both Vero and J774 cells compared with the wild-type (Fig. 9, Vero, $P<0.01$; J774, $P<0.05)$ at 8 h post-infection, whereas the mutant was similar in growth rate to the wild-type before this time point, indicating that IspC extends the existence of intracellular bacteria and promotes bacterial intracellular growth at the later infection stage.

\section{DISCUSSION}

In this work, we showed evidence that a cell wall-anchored peptidoglycan hydrolase (autolysin), IspC (Wang \& Lin, 2007), recognized also as the target of the humoral immune response to listerial infection ( $\mathrm{Yu}$ et al., 2007) and expressed as a minor autolysin in vitro (this study), is not important for cell division or separation during in vitro growth but is required for full virulence of $L$. monocytogenes. By in-frame-deleting the ispC gene, we have determined the effect of IspC deficiency on phenotypic characteristics of L. monocytogenes (i.e. in vitro growth, colony and cell morphologies, and biochemical properties), virulence of the bacterium in mouse and eukaryotic cell models of infection, and display of other surface proteins. 


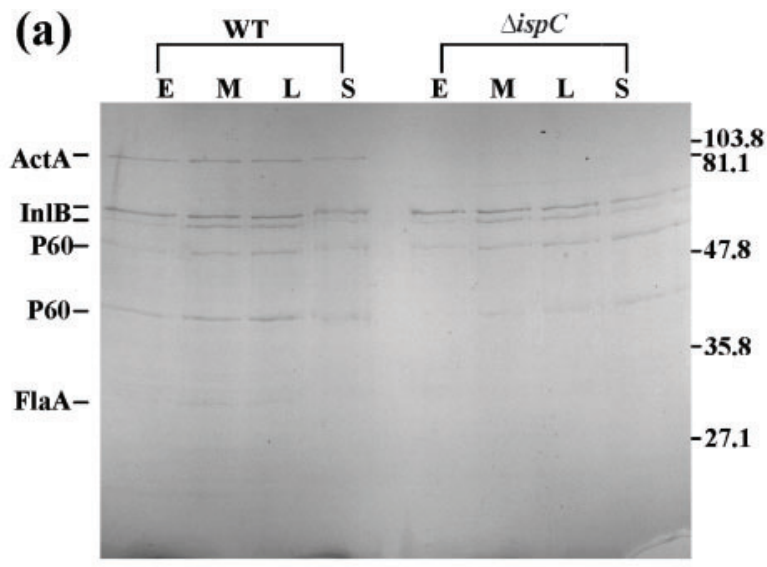

\section{(c)}
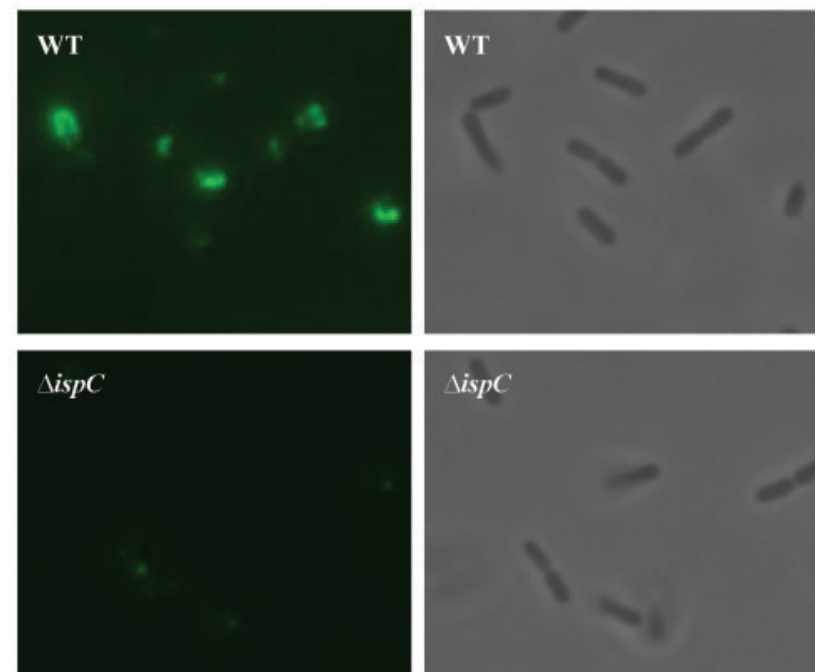

\section{$\triangle i s p C$}

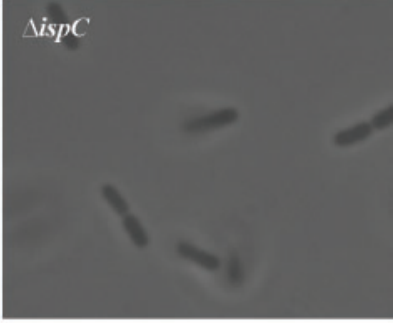

(b)
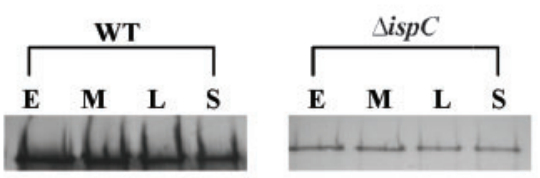

anti-Act A

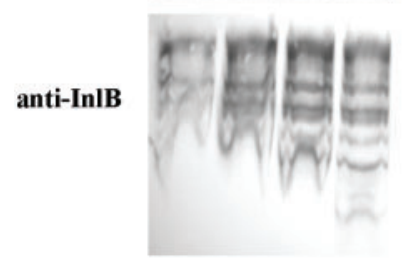

anti-InlA
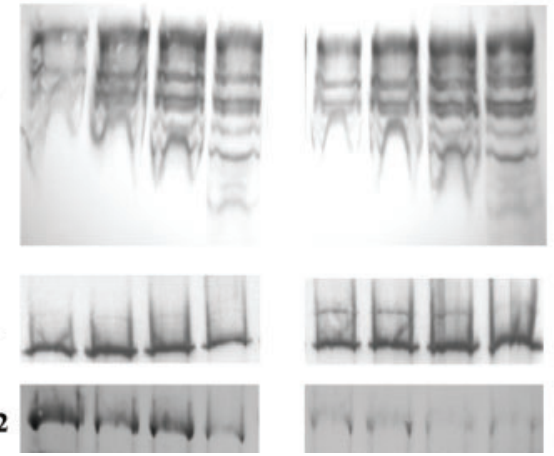

WT

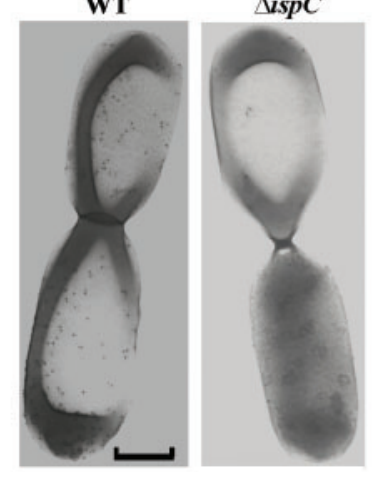

Fig. 6. Analysis of the cell surface proteins of the $\Delta i s p C$ mutant and the wild-type (WT) by SDS-PAGE and Western blotting. (a) Surface proteins were extracted from the mutant and wild-type strains at the early exponential (E), mid-exponential (M), lateexponential (L) and stationary (S) growth phases using $2 \times$ SDS-PAGE sample buffer and loaded into each lane for SDS-PAGE analysis. The proteins identified by in-gel digestion of excised bands with trypsin followed by MS analysis are shown on the left of the gel. Each lane was loaded with the surface proteins derived from bacterial cells equivalent to $7.5 \mathrm{ml}$ of culture with an $\mathrm{OD}_{620}$ of 0.5 . The molecular masses of protein standards (in $\mathrm{kDa}$ ) are indicated on the right. (b) The surface protein extracts from the mutant and wild-type strains were resolved by SDS-PAGE followed by Western blot analysis using rabbit anti-ActA, $-\operatorname{In|B},-\ln \mid \mathrm{A}$ and $-\operatorname{In|C} 2$ antibodies at a $1: 1000$ dilution. Surface proteins derived from bacterial cells equivalent to $1 \mathrm{ml}$ culture with an $\mathrm{OD}_{620}$ of 0.5 were loaded into each lane. (c) Detection of the surface expression of ActA protein of the mutant and wildtype bacteria at the mid-exponential growth phase by immunofluorescence staining (left-top and left-middle panels, immunofluorescence micrographs; right-top and right-middle panels, phase-contrast micrographs) and immunogold-labelling TEM (bottom panels), probed with rabbit anti-ActA antiserum. Bar, $0.2 \mu \mathrm{m}$.

The findings from these analyses and from the analysis of the binding to eukaryotic cells of the purified IspC and of its C-terminal CWBD (amino acids 198-774) fused to GFP (Wang \& Lin, 2007) have shed new light on our understanding of the molecular mechanisms by which a surface peptidoglycan hydrolase contributes to bacterial pathogenesis.

The in vivo study demonstrated a marked attenuation of virulence of the $\Delta i s p C$ mutant for mice. Similarly, in vivo studies with animal models (mice, rats and guinea pigs) of infection have shown that mutant strains defective in the synthesis of autolysins, including AtlE of Staph. epidermidis (Rupp et al., 2001), p60, Ami and Auto of L. monocytogenes (Cabanes et al., 2004; Milohanic et al., 2001; Pilgrim et al., 2003), and LytA of Strep. pneumoniae (Berry \& Paton, 2000), are less virulent than the wild-type strains. Colonization of liver and brain by L. monocytogenes was much more IspC-dependent than was that of spleen. The observed effect on virulence, as reflected by the impaired ability to colonize the target organs (liver and brain) of the $\triangle i s p C$ mutant, is unlikely to be attributable to the growth 

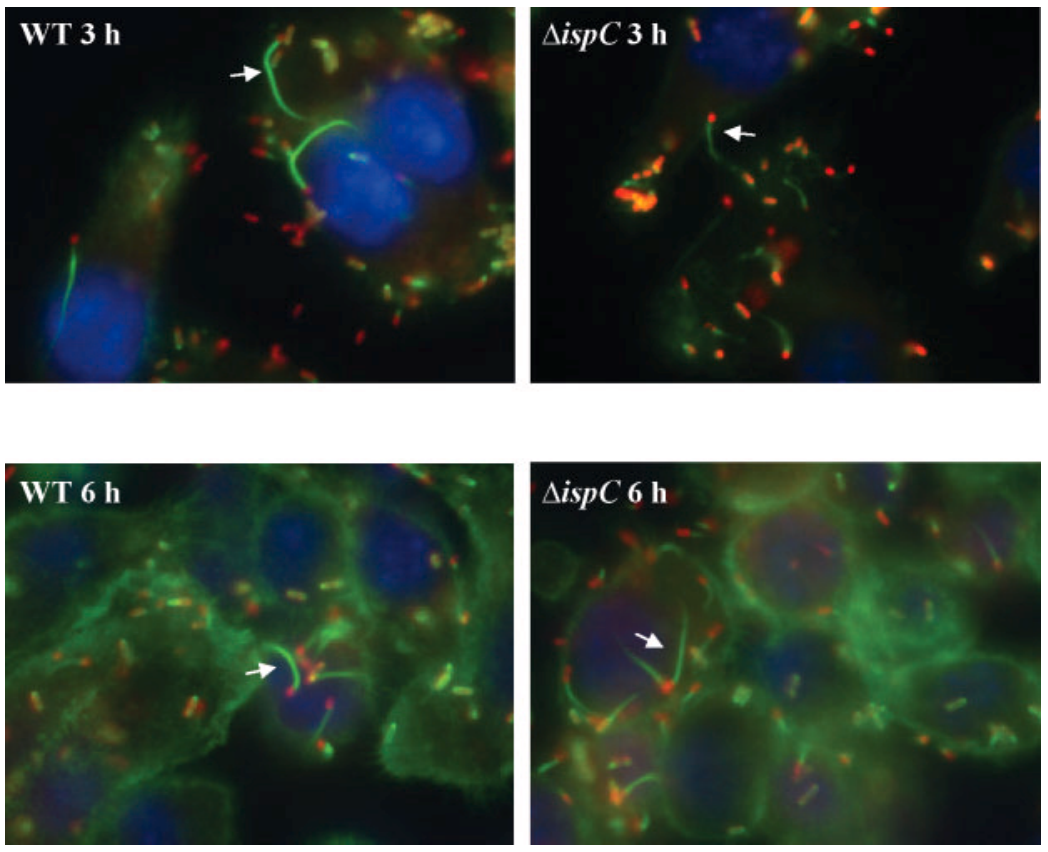

\begin{abstract}
Fig. 7. Detection of actin tail formation in mouse macrophage J774 cells following infection with the $\Delta i s p C$ mutant and the wild-type (WT). Actin tail formation promoted by ActA from the mutant and wild-type strains was examined at the early (3 h) and later $(6 \mathrm{~h})$ stages of $\mathbf{J 7 7 4}$ infection. The actin tails (green), bacteria (red) and nuclei (blue) were stained with Alexa Fluor 488 Phalloidin and rabbit anti-ActA antibody, followed by interaction with Alexa Fluor 647 goat anti-rabbit antibody and DAPI, respectively. The images were visualized with a fluorescence microscope equipped with a digital video camera. The difference in actin tail formation between the mutant and the wild-type is indicated with arrows.
\end{abstract}

rate, colony or cell morphology, or biochemical characteristics of the mutant strain, because in vitro the $\Delta i s p C$ mutant is similar in these aspects to the wild-type strain. These findings suggest that IspC does not function in cell division or separation during in vitro growth, for which other autolysins, p60 and MurA (NamA), have been shown to be necessary in L. monocytogenes (Carroll et al., 2003; Machata et al., 2005; Pilgrim et al., 2003). Therefore, IspC, deficiency of which is responsible for the observed attenuated virulence in mice, is involved in the establishment of L. monocytogenes infection in vivo.

This study has further characterized the $\Delta i s p C$ mutant in detail using a cell culture infection model employing various eukaryotic cell types that the bacterium normally encounters during in vivo infection, leading to a better understanding of the principal roles of IspC in pathogenesis. The results obtained from these experiments demonstrate that IspC significantly facilitates the infectious process of L. monocytogenes at multiple steps that are known to require several key virulence factors (VazquezBoland et al., 2001), such as internalins (InlA and InlB), responsible for bacterial entry into host cells, phospholipases (PlcA and $\mathrm{PlcB}$ ) and listeriolysin $\mathrm{O}$ (LLO), for escape from the phagosomes, and the actin polymerization (actin tail)-promoting protein ActA, for intracellular movement and cell-to-cell spread. The impaired capability of the
$\Delta i s p C$ mutant to adhere to Hep-G2, Vero and SCP cells but not to other eukaryotic cells (Caco-2, L132, Hela, HBMEC and J774) suggests that IspC functions in pathogenesis as an adhesin, mediating the attachment of the bacterium to certain eukaryotic cells, which presumably express unidentified receptor(s) specific for IspC. Localization of IspC on the cell surface (Wang \& Lin, 2007) meets the requirement for this protein to act as an adhesin. The adhesin nature of IspC is supported by the observation that purified IspC was capable of binding to SCP and Vero via its C-terminal CWBD, made of seven GW modules. The adhesive properties of other autolysins, including Aas of Staph. saprophyticus (Hell et al., 1998), AtlC of Staph. caprae (Allignet et al., 2002), AtlE of Staph. epidermidis (Heilmann et al., 1997) and Ami of L. monocytogenes (Milohanic et al., 2001), have been shown. Comparison of the adherence of the $\Delta i s p C$ mutant to various eukaryotic cells with the invasion of these cells by the mutant points to some interesting facts: (i) the mutant, which has an impaired ability to adhere to the cell lines Hep-G2, Vero and SCP, is less invasive to these cells; and (ii) the mutant strain, which has no reduction in its ability to adhere to Hela and HBMEC, is fully capable of invading these cells. One exception to this is that although the $\Delta i s p C$ mutant was capable of adhering to Caco-2 and L132, it showed impaired ability to invade these cells. These results indicate that IspC is necessary for entry of L. monocytogenes into 


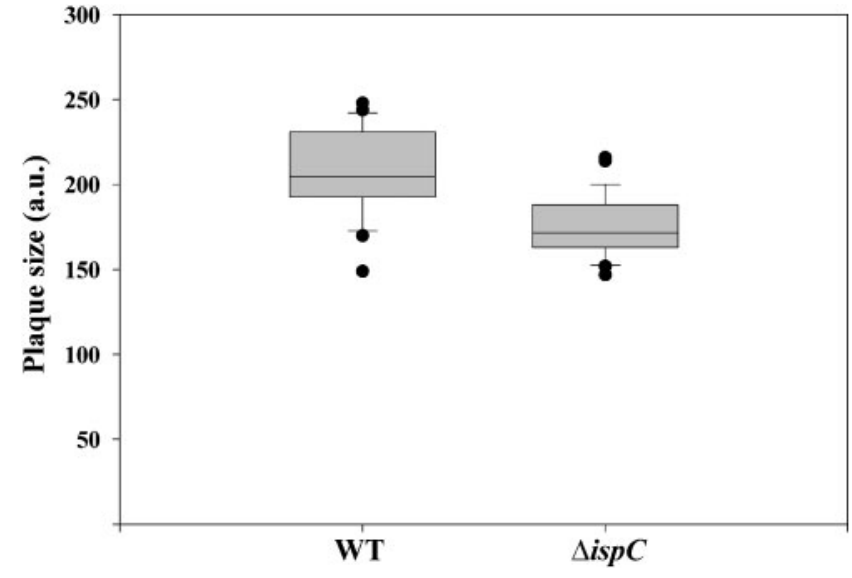

Fig. 8. Box plot comparison of the sizes of plaques formed from murine L2 cells infected with the $\triangle i s p C$ mutant and the wild-type (WT). At least 20 plaques were randomly selected for each strain for determination of their relative sizes (diameter in arbitrary units; a.u.) by analysing the plaque images with ImageJ software. The lower boundary of the box, the line within the box, and the upper boundary of the box correspond to the lower quartile (the 25th percentile), the median and the upper quartile (the 75th percentile), respectively. Error bars above and below the box mark the 90th and 10th percentiles. Solid circles represent the unusual observations (outliers) greater than the 90th percentile or less than the 10th percentile.

specific types of eukaryotic cells, although this may not be always dependent on IspC-mediated adhesion. Surprisingly, the autolysin Auto of L. monocytogenes, similar in domain organization to two other L. monocytogenes autolysins, Ami and IspC, with an affinity for certain eukaryotic cells (Milohanic et al., 2001; Wang \& Lin, 2007; this study), is not required for adhesion to, but is required for entry into, eukaryotic cells (Cabanes et al., 2004). It appears that the importance of a particular autolysin in L. monocytogenes pathogenesis is dependent on the cell type (epithelial cells, fibroblasts, hepatocytes, endothelial cells and macrophages) that the bacterium encounters during in vivo infection.

It was interesting to observe that adhesion to and invasion of SCP epithelial cells but not HBMEC by L. monocytogenes was dependent on the product of ispC. This is a novel finding with implications for the role that IspC plays in $L$. monocytogenes infection of the brain. There are two brain barriers: the cerebral capillary endothelium, as the barrier between the blood and the brain parenchyma; and the choroid plexus epithelium, as the barrier between blood and the cerebrospinal fluid (CSF) (Tuomanen, 1996). It has been shown that efficient invasion of HBMEC by L. monocytogenes depends on InlB (Greiffenberg et al., 1998). We have first used SCP cells to demonstrate that L. monocytogenes invades these cells in an IspC-dependent manner. This

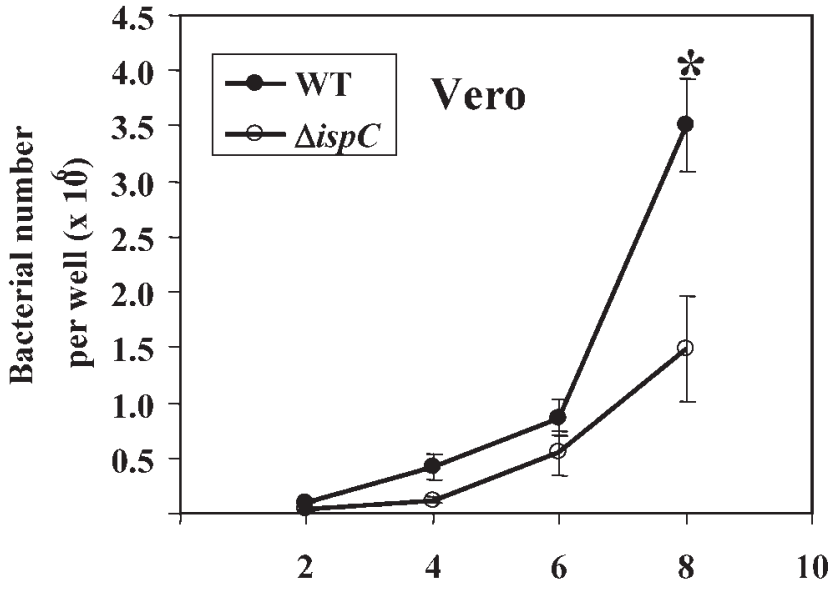

Time (h)

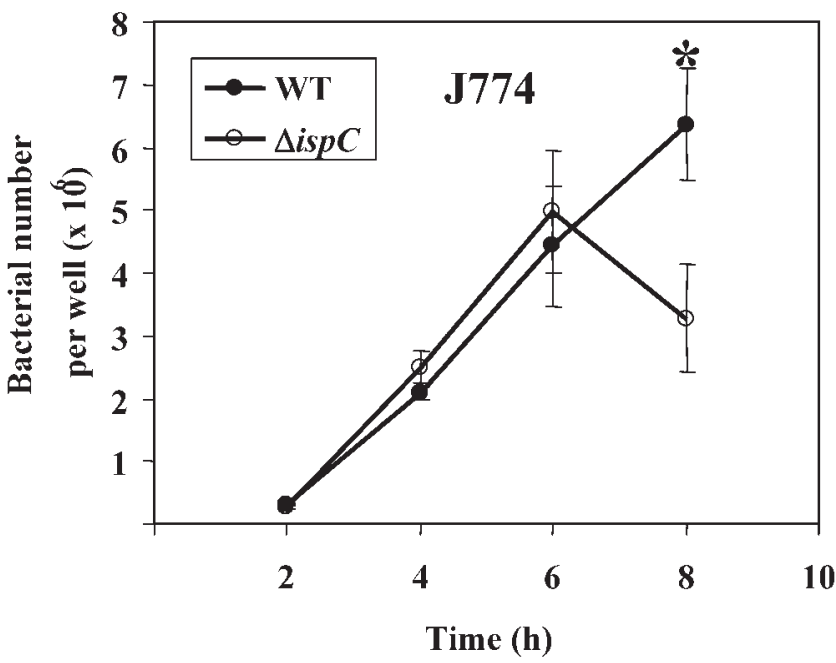

Fig. 9. Quantitative analysis of the intracellular growth of the $\triangle i s p C$ mutant in Vero and $\mathrm{J} 774$ cells. The intracellular growth assay (see Methods) was performed to assess bacterial intracellular growth. Mutant and wild-type (WT) bacteria were used to infect two representative cell lines (Vero and J774) at a predetermined m.o.i. for $1 \mathrm{~h}$ followed by further incubation with $100 \mu \mathrm{g}$ gentamicin $\mathrm{ml}^{-1}$. The numbers of intracellular viable bacteria were determined 2, 4, 6 and $8 \mathrm{~h}$ after addition of gentamicin as in Fig. 3, and are presented as the mean \pm SD $(n=3)$. *Statistically significant difference $(P<0.05)$.

suggests that when cultured human epithelial cells from the choroid plexus are not available, SCP cells are a good cell culture model for in vitro study of adhesion to and entry into the epithelial cells of the choroid plexus by L. monocytogenes, which has been shown to cause significant meningitis in sheep (Vandegraaff et al., 1981). Strong adhesion of $L$. monocytogenes to the choroid plexus was also observed in a mouse infection model (Schluter et al., 1996). Based on the observations reported here and elsewhere (Prats et al., 1992; Schluter et al., 1996), we propose a model for $L$. monocytogenes to breach the blood-brain barrier, causing 
meningitis or encephalitis. The entry of the bacterium into the epithelial cells of the choroid plexus is mediated by IspC via interaction with an unknown receptor; when within the epithelial cells, the bacterium undergoes cell-to-cell spread or enters into the CSF to undergo an extracellular phase prior to causing brain infection (encephalitis). Demonstration of the presence of L. monocytogenes in CSF (Brouwer et al., 2006, Schluter et al., 1996) supports the notion of $L$. monocytogenes undergoing an extracellular phase. Alternatively, L. monocytogenes could invade the microvascular endothelial cells through the interaction of InlB with a specific, as yet unidentified receptor, leading to encephalitis. The important but different functions of both IspC and InlB in brain pathogenesis caused by $L$. monocytogenes may be explained by the facts that both proteins contain the C-terminal CWBD made up of repeated GW modules, with variation in number and amino acid sequence for each protein (Braun et al., 1997; Wang \& Lin, 2007); this domain was shown to be responsible for interaction of IspC with SCP epithelial cells (this study), and presumably accounted for binding of InlB to HBMEC.

Formation of shorter and weaker actin tails during early infection of J774 cells with the $\Delta i s p C$ mutant and of smaller sizes of plaques following infection of L2 fibroblasts with the mutant, and significant reduction in growth of the mutant within Vero and J744 cells suggest that efficient intracellular movement, cell-to-cell spread, and intracellular survival of the bacterium necessarily depend on the expression of the is $p C$ gene. We observed a marked reduction in the amount of the surface protein ActA due to the absence of IspC during in vitro growth. This seems to suggest that IspC regulates the surface display of ActA, presumably through its autolytic activity, and thus promotes intracellular movement and cell-to-cell spread. However, the expression of ActA on the bacterial surface within the infected cells (J774) did not seem to be affected by lack of IspC expression in the $\Delta i s p C$ mutant (our unpublished data). Deletion of the $p 60$ gene from L. monocytogenes has been shown to affect the polarization of ActA on the bacterial surface, leading to loss of actinbased motility (Pilgrim et al., 2003). It may be speculated that the deletion of ispC alters the polarization of ActA on the surface of bacteria during the intracellular phase of growth, resulting in shorter and weaker actin tails in early stages of infection.

Using proteomic and immunological analysis, we have assessed the effect of IspC deficiency on the display of surface proteins. The reduced surface expression in the $\Delta i s p C$ mutant of ActA, a putative LPXTG motif-containing internalin, InlC2, and a flagellin-like protein homologous to the $30 \mathrm{kDa}$ flagellin FlaA with peptidoglycan hydrolase activity in L. monocytogenes EGD-e (Popowska \& Markiewicz, 2004) suggests that IspC may also be indirectly involved in pathogenesis, because these surface proteins were demonstrated to be, or were implicated as, virulence factors. In addition to the role of ActA in promoting the actin-based intra- and intercellular movement of
L. monocytogenes, this protein has been shown to mediate the attachment to and entry of the bacterium into eukaryotic cells (Alvarez-Dominguez et al., 1997). Although the role of $\mathrm{InlC2}$ in pathogenesis remains undefined, this protein may be required for bacterial survival under osmotic and/or stationary-phase stress (Dramsi et al., 1997; Kazmierczak et al., 2003). The FlaA protein, a structural component of flagella, is capable of facilitating the initial association of the bacterium with and effective invasion of epithelial cells (Dons et al., 2004), and enhances L. monocytogenes infectivity after ingestion (O'Neil \& Marquis, 2006). Thus, the attenuated virulence of the $\Delta i s p C$ mutant observed with mouse and cell culture infection models may be partly due to the reduced surface expression or display of other known or putative virulence factors. It is unlikely that the C-terminal region of IspC, which is made up of seven GW modules and functions to anchor the protein to the cell wall (Wang \& Lin, 2007), is responsible for maintaining the proper display of other surface proteins. We propose that the autolytic activity conferred by the N-terminal catalytic domain of IspC (Wang \& Lin, 2007) breaks the chemical bonds within cell wall peptidoglycan to alter the surface properties (e.g. structure, charge and surface modification), as has been shown for p60 of L. monocytogenes (Pilgrim et al., 2003) and Atl of Staph. aureus (Takahashi et al., 2002), providing the cell wall architecture necessary for the proper polarization or display of virulence factors such as ActA, thereby allowing their function. This is also supported by our unpublished observation that the mutant strain was harder to disrupt by physical (ultrasonication) or enzymic (lysozyme digestion) methods than the wild-type. The inspection of proteins that exhibited a reduced surface expression (ActA, InlC2 and a FlaA homologue) and those that were unaffected (InlB, InlA and p60) appears to indicate that the influence on the display of surface proteins of IspC is independent of a particular surfacetargeting mechanism, because various surface-targeting mechanisms (Bierne \& Cossart, 2007; Cabanes et al., 2002) such as the LPXTG motif in InlA and InlC2, a C-terminal hydrophobic domain in ActA, a C-terminal domain consisting of repeated GW modules in InlB, and the LysM domain in $\mathrm{p} 60$, are employed in these proteins. The indirect role of IspC in virulence by promoting the surface display of other virulence factors is in contrast to the findings that the autolysins Auto and p60 of L. monocytogenes do not alter the expression of other major virulence factors such as InlA, InlB, ActA and LLO (Cabanes et al., 2004; Pilgrim et al., 2003). Thus, the present study is the first to demonstrate a dual role for a minor $L$. monocytogenes autolysin in virulence.

\section{ACKNOWLEDGEMENTS}

We acknowledge B. Phipps-Todd for technical support in electron microscopy, M. Wiedmann and E. D. Fortes (Department of Food Science, Cornell University, NY, USA) for useful advice in plaque assay, and the Small Animal Colony (Canadian Food Inspection 
Agency, Ottawa Laboratory, Fallowfield) for mouse infection experiments. The rabbit anti-InlA and -InlB antibodies were kindly provided by J. Wehland (Technical University of Braunschweig, Braunschweig, Germany) and rabbit anti-ActA antibody by M. Kuhn [Biocenter (Microbiology), University of Würzburg, Würzburg, Germany]. We also thank H. McRae, H. Dan and K. Coulombe for their critical reading of the manuscript.

\section{REFERENCES}

Allignet, J., England, P., Old, I. \& El Solh, N. (2002). Several regions of the repeat domain of the Staphylococcus caprae autolysin, AtlC, are involved in fibronectin binding. FEMS Microbiol Lett 213, 193-197.

Alvarez-Dominguez, C., Vazquez-Boland, J. A., Carrasco-Marin, E., Lopez-Mato, P. \& Leyva-Cobian, F. (1997). Host cell heparan sulfate proteoglycans mediate attachment and entry of Listeria monocytogenes, and the listerial surface protein ActA is involved in heparan sulfate receptor recognition. Infect Immun 65, 78-88.

Bergmann, B., Raffelsbauer, D., Kuhn, M., Goetz, M., Hom, S. \& Goebel, W. (2002). InlA- but not InlB-mediated internalization of Listeria monocytogenes by non-phagocytic mammalian cells needs the support of other internalins. Mol Microbiol 43, 557-570.

Berry, A. M. \& Paton, J. C. (2000). Additive attenuation of virulence of Streptococcus pneumoniae by mutation of the genes encoding pneumolysin and other putative pneumococcal virulence proteins. Infect Immun 68, 133-140.

Berry, A. M., Lock, R. A., Hansman, D. \& Paton, J. C. (1989). Contribution of autolysin to virulence of Streptococcus pneumoniae. Infect Immun 57, 2324-2330.

Bierne, H. \& Cossart, P. (2007). Listeria monocytogenes surface proteins: from genome predictions to function. Microbiol Mol Biol Rev 71, 377-397.

Braun, L., Dramsi, S., Dehoux, P., Bierne, H., Lindahl, G. \& Cossart, P. (1997). InlB: an invasion protein of Listeria monocytogenes with a novel type of surface association. Mol Microbiol 25, 285-294.

Brouwer, M. C., van de Beek, D., Heckenberg, S. G., Spanjaard, L. \& de Gans, J. (2006). Community-acquired Listeria monocytogenes meningitis in adults. Clin Infect Dis 43, 1233-1238.

Cabanes, D, Dehoux, P., Dussurget, O., Frangeul, L. \& Cossart, P. (2002). Surface proteins and the pathogenic potential of Listeria monocytogenes. Trends Microbiol 10, 238-245.

Cabanes, D., Dussurget, O., Dehoux, P. \& Cossart, P. (2004). Auto, a surface associated autolysin of Listeria monocytogenes required for entry into eukaryotic cells and virulence. Mol Microbiol 51, 16011614.

Canvin, J. R., Marvin, A. P., Sivakumaran, M., Paton, J. C., Boulnois, G. J., Andrew, P. W. \& Mitchell, T. J. (1995). The role of pneumolysin and autolysin in the pathology of pneumonia and septicemia in mice infected with a type 2 pneumococcus. J Infect Dis 172, 119-123.

Carroll, S. A., Hain, T., Technow, U., Darji, A., Pashalidis, P., Joseph, S. W. \& Chakraborty, T. (2003). Identification and characterization of a peptidoglycan hydrolase, MurA, of Listeria monocytogenes, a muramidase needed for cell separation. J Bacteriol 185, 6801-6808.

Dons, L., Eriksson, E., Jin, Y., Rottenberg, M. E., Kristensson, K., Larsen, C. N., Bresciani, J. \& Olsen, J. E. (2004). Role of flagellin and the two-component $\mathrm{CheA} / \mathrm{CheY}$ system of Listeria monocytogenes in host cell invasion and virulence. Infect Immun 72, 3237-3244.

Dramsi, S., Dehoux, P., Lebrun, M., Goossens, P. L. \& Cossart, P. (1997). Identification of four new members of the internalin multigene family of Listeria monocytogenes EGD. Infect Immun 65, $1615-1625$.
Greiffenberg, L., Goebel, W., Kim, K. S., Weiglein, I., Bubert, A., Engelbrecht, F., Stins, M. \& Kuhn, M. (1998). Interaction of Listeria monocytogenes with human brain microvascular endothelial cells: InlB-dependent invasion, long-term intracellular growth, and spread from macrophages to endothelial cells. Infect Immun 66, 5260-5267.

Heilmann, C., Hussain, M., Peters, G. \& Gotz, F. (1997). Evidence for autolysin-mediated primary attachment of Staphylococcus epidermidis to a polystyrene surface. Mol Microbiol 24, 1013-1024.

Hell, W., Meyer, H. G. \& Gatermann, S. G. (1998). Cloning of aas, a gene encoding a Staphylococcus saprophyticus surface protein with adhesive and autolytic properties. Mol Microbiol 29, 871-881.

Jedrzejas, M. J. (2001). Pneumococcal virulence factors: structure and function. Microbiol Mol Biol Rev 65, 187-207.

Kazmierczak, M. J., Mithoe, S. C., Boor, K. J. \& Wiedmann, M. (2003). Listeria monocytogenes $\sigma^{\mathrm{B}}$ regulates stress response and virulence functions. J Bacteriol 185, 5722-5734.

Lenz, L. L., Mohammadi, S., Geissler, A. \& Portnoy, D. A. (2003). SecA2-dependent secretion of autolytic enzymes promotes Listeria monocytogenes pathogenesis. Proc Natl Acad Sci U S A 100, 1243212437.

Lock, R. A., Hansman, D. \& Paton, J. C. (1992). Comparative efficacy of autolysin and pneumolysin as immunogens protecting mice against infection by Streptococcus pneumoniae. Microb Pathog 12, 137-143.

MacFaddin, J. F. (2000). Biochemical Tests for Identification of Medical Bacteria, 3rd edn. New York: Lippincott Williams \& Wilkins.

Machata, S., Hain, T., Rohde, M. \& Chakraborty, T. (2005). Simultaneous deficiency of both MurA and p60 proteins generates a rough phenotype in Listeria monocytogenes. J Bacteriol 187, 83858394.

Mani, N., Baddour, L. M., Offutt, D. Q., Vijaranakul, U., Nadakavukaren, M. J. \& Jayaswal, R. K. (1994). Autolysis-defective mutant of Staphylococcus aureus: pathological considerations, genetic mapping, and electron microscopic studies. Infect Immun 62, 14061409.

Milohanic, E., Jonquieres, R., Cossart, P., Berche, P. \& Gaillard, J. L. (2001). The autolysin Ami contributes to the adhesion of Listeria monocytogenes to eukaryotic cells via its cell wall anchor. Mol Microbiol 39, 1212-1224.

O'Neil, H. S. \& Marquis, H. (2006). Listeria monocytogenes flagella are used for motility, not as adhesins, to increase host cell invasion. Infect Immun 74, 6675-6681.

Park, S. F. \& Stewart, G. S. (1990). High-efficiency transformation of Listeria monocytogenes by electroporation of penicillin-treated cells. Gene 94, 129-132.

Pilgrim, S., Kolb-Maurer, A., Gentschev, I., Goebel, W. \& Kuhn, M. (2003). Deletion of the gene encoding p60 in Listeria monocytogenes leads to abnormal cell division and loss of actin-based motility. Infect Immun 71, 3473-3484.

Popowska, M. (2004). Analysis of the peptidoglycan hydrolases of Listeria monocytogenes: multiple enzymes with multiple functions. Pol J Microbiol 53 (suppl.), 29-34.

Popowska, M. \& Markiewicz, Z. (2004). Murein-hydrolyzing activity of flagellin FlaA of Listeria monocytogenes. Pol J Microbiol 53, 237-241.

Prats, N., Briones, V., Blanco, M. M., Altimira, J., Ramos, J. A., Dominguez, L. \& Marco, A. (1992). Choroiditis and meningitis in experimental murine infection with Listeria monocytogenes. Eur J Clin Microbiol Infect Dis 11, 744-747.

Rowan, N. J., Candlish, A. A., Bubert, A., Anderson, J. G., Kramer, K. \& McLauchlin, J. (2000). Virulent rough filaments of Listeria monocytogenes from clinical and food samples secreting wild-type levels of cell-free p60 protein. J Clin Microbiol 38, 2643-2648. 
Rupp, M. E., Ulphani, J. S., Fey, P. D., Bartscht, K. \& Mack, D. (1999). Characterization of the importance of polysaccharide intercellular adhesin/hemagglutinin of Staphylococcus epidermidis in the pathogenesis of biomaterial-based infection in a mouse foreign body infection model. Infect Immun 67, 2627-2632.

Rupp, M. E., Fey, P. D., Heilmann, C. \& Gotz, F. (2001). Characterization of the importance of Staphylococcus epidermidis autolysin and polysaccharide intercellular adhesin in the pathogenesis of intravascular catheter-associated infection in a rat model. J Infect Dis 183, 1038-1042.

Schaferkordt, S., Domann, E. \& Chakraborty, T. (1998). Molecular approaches for the study of Listeria. In Bacterial Pathogenesis (Methods in Microbiology no. 27) pp. 421-431. Edited by P. Williams, J. Ketley \& G. Salmond. San Diego, CA: Academic Press.

Schluter, D., Chahoud, S., Lassmann, H., Schumann, A., Hof, H. \& Deckert-Schluter, M. (1996). Intracerebral targets and immunomodulation of murine Listeria monocytogenes meningoencephalitis. J Neuropathol Exp Neurol 55, 14-24.

Shockman, G. D. \& Holtje, J.-V. (1994). Microbial peptidoglycan (murein) hydrolases. In Bacterial Cell Wall, pp. 131-166. Edited by J.-M. Ghuysen \& R. Hakenbeck. Amsterdam: Elsevier.

Smith, T. J., Blackman, S. A. \& Foster, S. J. (2000). Autolysins of Bacillus subtilis: multiple enzymes with multiple functions. Microbiology 146, 249-262.

Takahashi, J., Komatsuzawa, H., Yamada, S., Nishida, T. Labischinski, H., Fujiwara, T., Ohara, M., Yamagishi, J. \& Sugai, M. (2002). Molecular characterization of an atl null mutant of Staphylococcus aureus. Microbiol Immunol 46, 601-612.
Tuomanen, E. (1996). Entry of pathogens into the central nervous system. FEMS Microbiol Rev 18, 289-299.

Tuomanen, E. I. (2000). Pathogenesis of pneumococcal inflammation: otitis media. Vaccine 19 (suppl. 1), S38-S40.

Vandegraaff, R., Borland, N. A. \& Browning, J. W. (1981). An outbreak of listerial meningo-encephalitis in sheep. Aust Vet J 57, 94-96.

Vasilescu, J., Smith, J. C., Ethier, M. \& Figeys, D. (2005). Proteomic analysis of ubiquitinated proteins from human MCF-7 breast cancer cells by immunoaffinity purification and mass spectrometry. $J$ Proteome Res 4, 2192-2200.

Vazquez-Boland, J. A., Kuhn, M., Berche, P., Chakraborty, T., Dominguez-Bernal, G., Goebel, W., Gonzalez-Zorn, B., Wehland, J. \& Kreft, J. (2001). Listeria pathogenesis and molecular virulence determinants. Clin Microbiol Rev 14, 584-640.

Wang, L. \& Lin, M. (2007). Identification of IspC, an 86-kilodalton protein target of humoral immune response to infection with Listeria monocytogenes serotype $4 \mathrm{~b}$, as a novel surface autolysin. J Bacteriol 189, 2046-2054.

Wang, L., Walrond, L., Cyr, T. D. \& Lin, M. (2007). A novel surface autolysin of Listeria monocytogenes serotype $4 \mathrm{~b}$, IspC, contains a 23residue $\mathrm{N}$-terminal signal peptide being processed in E. coli. Biochem Biophys Res Commun 354, 403-408.

Yu, W. L., Dan, H. \& Lin, M. (2007). Novel protein targets of humoral immune response to Listeria monocytogenes infection in rabbits. J Med Microbiol 56, 888-895.

Edited by: H. Ingmer 